\title{
Maximum Principle for Totally Umbilical Null Hypersurfaces and Time-dependent Null Horizons
}

\author{
K. L. Duggal ${ }^{1}$ \\ ${ }^{1}$ Department of Mathematics and Statistics, University of Windsor, Windsor, Ontario N9B3P4, Canada \\ Correspondence: K. L. Duggal, Department of Mathematics and Statistics, University of Windsor, Windsor, On- \\ tario N9B3P4, Canada. E-mail: yq8@uwindsor.ca
}

Received: January 9, 2015 Accepted: January 31, 2015 Online Published: February 22, 2015

doi:10.5539/jmr.v7n1p88 ～URL: http://dx.doi.org/10.5539/jmr.v7n1p88

\begin{abstract}
In this paper we modify the maximum principal of (Galloway, 2000) for totally geodesic null hypersurfaces by proving a geometric maximum principle which obeys mean curvature inequalities of a family of totally umbilical null hypersurfaces of a spacetime manifold (Theorem 6). As a physical interpretation we show that, in particular, for a prescribed class of spacetimes the geometric inequality of the Theorem 6 for totally umbilical null hypersurfaces is valid as well as it establishes a link with Galloway's vanishing mean curvature totally geodesic null hypersurfaces that arise most naturally in general relativity, such as black hole event horizons (Theorem 7).
\end{abstract}

Keywords: maximum principle, totally umbilical null hypersurfaces, mean curvature, null horizons

Subject Clasification: Primary: 53C40, 53C50, 83C57 Secondary: 53C12, 53C22

\section{Introduction}

Recall that (Eschenburg, 1989) proved following result on the maximum principle for hypersurfaces (also valid for hypersurfaces of Lorentzian manifolds).

Theorem 1. Let $\Sigma_{+}$and $\Sigma_{-}$be disjoint open domains with spacelike connected $C^{2}$-boundaries having a point in common. If the mean curvatures $\theta_{+}$of $\partial \Sigma_{+}$and $\theta_{-}$of $\partial \Sigma_{-}$satisfy

$$
\theta_{-} \leq-a \quad, \quad \theta_{+} \leq a
$$

for some real number $a$, then $\partial \Sigma_{-}=\partial \Sigma_{+}$, and $-\theta_{-}=\theta_{+}=a$.

Extending above result, (Anderson, Galloway \& Howard, 1998) proved a geometric version of maximum principle for rough $\left(C^{0}\right)$ spacelike hypersurfaces of Lorentzian manifolds. Then, (Galloway, 2000) proved the following anologous result for smooth null hypersurfaces restricted to the zero mean curvature case and suitable to asymptotically flat spacetimes.

Theorem 2. Let $\Sigma_{1}$ and $\Sigma_{2}$ be smooth null hypersufaces in a spacetime manifold M. Suppose,

(1) $\Sigma_{1}$ and $\Sigma_{2}$ meet at $p \in M$ and $\Sigma_{2}$ lies to the future side of $\Sigma_{1}$ near $p$

(2) the null mean curvature scalars $\theta_{1}$ of $\Sigma_{1}$, and $\theta_{2}$ of $\Sigma_{2}$, satisfy, $\theta_{2} \leq 0 \leq \theta_{1}$.

Then $\Sigma_{1}$ and $\Sigma_{2}$ coincide near $p$ and this common null hypersurface has mean curvature $\theta=0$.

The proof is based on well-known classical result of (Alexandrov, 1954) on strong maximum principle (see Theorem 5) for second order quasi-linear elliptic PDE's. Galloway also discussed the $C^{0}$ version of above theorem since null hypersurfaces have been used in general relativity as models of black hole event and Cauchy horizons (Hawking \& Ellis, 1973) of asymptotically flat spacetimes, which are $C^{0}$ but in general not $C^{1}$. He further proved that if the spacetime $M$ obeys the null energy condition, $\operatorname{Ric}(X, X) \geq 0 \forall$ null vectors $X$, then, the common null hypersurface is totally geodesic in $M$. 
Since the Eschenburg's maximum principle holds for some real number $a$ (not restricted to $a=0$ ) in equality $-\theta_{-}=\theta_{+}=a$, it is quite reasonable to modify the condition (2) of Galloway's Theorem 2 such that, in general, $\theta_{2} \leq$ $\theta \leq \theta_{1}$ for some mean curvature $\theta$ of a time-dependent null hypersurface, say $\Sigma$, which may, in particular, evolve into a time-independent null hypersurface with vanishing mean curvature. The motivation for such modification is two fold: Firstly, since Galloway's Theorem 2 was designed for totally geodesic null hypersurfaces, we use totally umbilical geometry of null hypersurfaces of spacetimes which are not necessarily only asymptotically flat spacetimes. Secondly, for physical use our modified geometric maximum principle (see Theorems 6 and 7) is a step further towards the ongoing study of time-dependent null hypersurfaces of spacetimes and, in particular, some related to black hole event and isolated horizons. For details on this ongoing study, we refer two papers of (Sultana \& Dyer, 2004, 2005) and two papers of (Duggal, 2012, 2014). Also, we refer (Hawking, 1972), and (Ashtekar, Beetle \& Fairhurst, 1999) for basic information on black hole event and isolated horizons, respectively.

\section{Method}

In this section we brief on some methods needed to prove our two main results of next section. Recall that a hypersurface $(\Sigma, h)$ of spacetime manifold $(M, g)$ is null if there exists a non-vanishing null vector field $\ell$ of $T(\Sigma)$ which is orthogonal (with respect to $h$ ) to all vector fields in $T(\Sigma)$, that is,

$$
h(\ell, X)=0, \quad \forall X \in T(\Sigma),
$$

where $h$ is the degenerate metric of $\Sigma$. In this paper we assume that the null normal $\ell$ is future directed and it is not entirely in $\Sigma$, but, is defined in some open subset of $M$ around $\Sigma$. A simple way to take this extended $\ell$ is to consider a foliation of $M$ (in the vicinity of $\Sigma$ ) by a family $\left(\Sigma_{u}\right)$ so that each $\ell_{u}$ is in the part of $M$ foliated by this family such that at each point in this region, $\ell_{u}$ is a null normal to $\left(\Sigma_{u}\right)$ for some value of $u$. Denote by $\left(h_{u}\right)$ the respective family of degenerate metrics. Although the family $\left(\Sigma_{u}\right)$ is not unique, for our purpose we can manage (with some reasonable condition(s)) to involve only those quantities which are independent of the choice of the foliation $\left(\Sigma_{u}\right)$ once evaluated at $u=$ constant. For simplicity, we consider $(\Sigma, h)$ as a member of the family $\left(\left(\Sigma_{u}\right),\left(h_{u}\right)\right)$ and its respective metric $h$ for some value of $u$, with the understanding that the results are same for any other member. This will allow us to use the following features of the intrinsic geometry of null hypersurfaces needed in this paper.

(a) It will permit to well-define the spacetime covariant derivative $\nabla \ell$ where $\nabla$ denotes the Levi-Civita connection on $M$.

(b) The "bending" of each $\Sigma$ in $M$ (with respect to each $\ell$ ) is described by the Weingarten map:

$$
\mathcal{W}_{\ell}: T_{p}(\Sigma) \rightarrow T_{p}(\Sigma), \quad X \rightarrow \nabla_{X} \ell .
$$

$\mathcal{W}_{\ell}$ associates each $X$ of $\Sigma$ the variation of $\ell$ along $X$, with respect to the spacetime connection $\nabla$.

(c) The second fundamental form, say $B_{\ell}$ with respect to null normal $\ell$ of $\Sigma$ is the symmetric bilinear form and is related to the Weingarten map by

$$
B_{\ell}(X, Y)=h\left(\mathcal{W}_{\ell} X, Y\right)=h\left(\nabla_{X} \ell, Y\right) .
$$

$B_{\ell}(X, \ell)=0$ for any null normal $\ell$ and for any $X \in T(\Sigma)$ implies that $B_{\ell}$ has the same $\ell$ degeneracy as that of the induced metric $h$. From (2.2) and $B_{\ell}$ symmetric implies that

$$
B_{\ell}(X, Y)=\frac{1}{2} £_{\ell} h(X, Y), \quad \forall X, Y \in T \Sigma .
$$

If $B_{\ell}$ is conformally equivalent to the metric $h$, then, we say that $(\Sigma, h)$ is totally umbilical in $M$ if and only if there is a smooth function $f$ on $\Sigma$ such that

$$
B_{\ell}(X, Y)=f h(X, Y), \quad \forall X, Y \in T(\Sigma) .
$$

A well-known example is the family of null cones of $M$ (Duggal, 2012), all of which are totally umbilical. We refer pages $106-138$ of (Duggal \& Bejancu, 1996) for the definition (2.4) and the basic information on totally umbilical null (also called lightlike) hypersurfaces.

Proposition 3. (Duggal, 2014) Let $F=\left(\left(\Sigma_{u}\right),\left(h_{u}\right)\right)$ be a family of null hypersurfaces of a spacetime manifold $(M, g)$. Then each member $(\Sigma, h)$ of the family $F$ is totally umbilical if and only if its null normal $\ell$ is a conformal Killing vector with respect to the degenerate metric $h$. 
Based on above result, it follows from the Equations (2.3) and (2.4) that $\Sigma$ is totally umbilical if and only if $£_{\ell} h=2 f h$ on $\Sigma$, that is, $\ell$ is conformal Killing vector field (CKV) of the degenerate metric $h$ with conformal function $2 f$. Note that it is not necessary that $\ell$ is a CKV field of the full metric $g$. In particular, $\Sigma$ is called totally geodesic if and only if $B_{\ell}$ vanishes, i.e., if and only if $f$ vanishes on $\Sigma$. For this case, $\ell$ will be a Killing vector (KV) field on $\Sigma$. See page 88 of (Duggal \& Bejancu, 1996) for the definition of totally geodesic null (lightlike) hypersurfaces.

In this paper, we let $(M, g)$ be an $n$-dimensional spacetime manifold evolved from a spacelike hypersurface $H_{t}$ at a coordinate time $t$ to another spacelike hypersurface $H_{t+d t}$ at coordinate time $t+d t$ whose metric $g$ is given by

$$
d s_{\mid g}^{2}=g_{i j} d x^{i} d x^{j}=\left(-\lambda^{2}+|U|^{2}\right) d t^{2}+2 \gamma_{a b} U^{a} d x^{b} d t+\gamma_{a b} d x^{a} d x^{b}
$$

where $x^{0}=t$, and $x^{a}(a=1, \cdots, n-1)$ are spatial coordinates of the hypersurface $H_{t}$ with $\gamma_{a b}$ its $(n-1)$-metric induced from $g, \lambda=\lambda\left(t, x^{1}, \cdots, x^{n-1}\right)$ is the lapse function and $U$ is a spacelike shift vector (Arnowitt, Deser $\&$ Misner, 1962). The coordinate time vector $\mathbf{t}=\frac{\partial}{\partial t}$ is such that $\mathrm{g}(\mathrm{dt}, \mathbf{t})=1$. This choice of spacetime is suitable for the proof of our two main theorems. Also, in Section 3 see some references on the physical use of this metric. We write

$$
\mathbf{t}=\lambda \mathbf{n}+U, \quad \text { with } \quad \mathbf{n} \cdot U=0,
$$

where $\mathbf{n}$ is the future timelike unit vector field. In general, each spacelike hypersurface $H_{t}$ intersects a null hypersurface say $\Sigma$ on some $(n-2)$-dimensional submanifold $\mathbf{S}_{t}$, that is, $\mathbf{S}_{t}=\Sigma \cap H_{t}$. Consider a family $F=\left(\left(\Sigma_{u}\right),\left(h_{u}\right)\right)$ with $\mathbf{S}_{t, u}=\Sigma_{u} \cap H_{t}$, where $\mathbf{S}_{t, u}, u=$ constant, is an element of the family $\left(\mathbf{S}_{t, u}\right)$ and $\left(h_{u}\right)$ the respective family of degenerate metrics of $\left(\Sigma_{u}\right)$. As explained before, we let $\ell$ be the future directed null normal which is not entirely in a member $\Sigma$ of the family $F$, but, is defined in some open subset of $M$ around that $\Sigma$. Let $\mathbf{s} \in H_{t}$ be a unit vector field normal to $\mathbf{S}_{t}$ defined in some open neighborhood of $\Sigma$. Taking $\left(\mathbf{S}_{t, u}\right)$ a foliation of $\left(\Sigma_{u}\right)$, the coordinate $t$ can be used as a non-affine parameter along each null geodesic generating each $\Sigma_{u}$. We normalize each member $\ell$ of $F$ such that it is tangent vector associated with this parameterization of the null generators, i.e., $\ell^{i}=\frac{d x^{i}}{d t}$. This means that $\ell$ is a vector field "dual" to the 1 -form $d t$. Equivalently, the function $t$ can be regarded as a coordinate compatible with $\ell$, i.e., $g(d t, \ell)=\nabla_{\ell} t=1$. Based on this, we choose the following normalization of each $\ell_{u} \in\left(\ell_{u}\right)$ for some value of $u$.

$$
\ell_{u}=\left(\mathbf{n}_{u}+\mathbf{s}_{u}\right), \quad \text { where } \quad \mathbf{s}_{u} \cdot s_{u}=1, \quad \mathbf{x} \in T_{p}\left(\mathbf{S}_{t}\right) \Leftrightarrow \mathbf{s}_{u} \cdot \mathbf{x}=0,
$$

which implies that each $\ell_{u}$ is tangent to each member of $F$ and it has the property of Lie dragging the family of surfaces $\left(\mathbf{S}_{t, u}\right)$. Then, we define a transversal vector field $\mathbf{k}_{u}$ of $T_{p} M$ not belonging to $F$ expressed as another suitable linear combination of $\mathbf{n}_{u}$ and $\mathbf{s}_{u}$ such that it represents the light rays emitted in the opposite direction, called the ingoing direction, satisfying:

$$
g\left(\ell_{u}, \mathbf{k}_{u}\right)=-1, \quad \mathbf{k}_{u}=\frac{1}{2}\left(\mathbf{n}_{u}-\mathbf{s}_{u}\right) .
$$

We say that two null normals $\ell_{i}$ and $\ell_{j}$ of $\Sigma_{i}$ and $\Sigma_{j}$ belong to the same equivalence class $\left[\ell_{u}\right]$ if $\ell_{j}=c \ell_{i}$ for some positive constant $c$. Then, there is another corresponding $\mathbf{k}_{j}=(1 / c) \mathbf{k}_{i}$ satisfying (2.8).

\section{Results}

We work with an element $(\Sigma, h, \ell, \mathbf{k})$ and an element $\left(\mathbf{S}_{t}, \hat{h}\right)$ of the two families $F$ and $\left(\mathbf{S}_{t, u}\right)$, respectively, where $\hat{h}$ is the induced Riemannian metric of $\mathbf{S}_{t}$. Using the spacelike normal $\mathbf{s}$ and timelike normal $\mathbf{n}$ it is easy to see that

$$
\hat{h}=\gamma-\underline{\mathbf{s}} \otimes \underline{\mathbf{s}}=g+\underline{\mathbf{n}} \otimes \underline{\mathbf{n}}-\underline{\mathbf{s}} \otimes \underline{\mathbf{s}},
$$

where underline is a symbol for the 1 -form. Consider the projection along $\ell$ on $T_{p}\left(\mathbf{S}_{t}\right)$ defined by

$$
\mathbf{P}: T_{p} \Sigma \rightarrow T_{p}\left(\mathbf{S}_{t}\right), \quad X \rightarrow \mathbf{x}=X-a \ell, \quad a \text { is a real number. }
$$

Proposition 4. The positive definite metric $\hat{h}$ induced by $g$ on $T_{p}\left(\mathbf{S}_{t}\right)$ coincides with the degenerate metric $h$ induced by $g$ on $T_{p}(\Sigma)$.

Proof. Let $\mathbf{x}$ and $\mathbf{y}$ be the projections along their respective pair of vectors $(X, Y)$ in $T_{p}(\Sigma)$. Then, as per Equation (3.2) we have the unique decompositions

$$
X=\mathbf{x}+a \ell, \quad Y=\mathbf{y}+b \ell,
$$


for two real numbers $a$ and $b$. Using $\mathbf{n} . \mathbf{x}=\mathbf{n . y}=\mathbf{s . x}=\mathbf{s . y}=0$ in the righthand side of the relation (3.1) it is straightforward to get $\hat{h}(X, Y)=g(X, Y)$. Thus, $\hat{h}$ and $g$ coincide on $T_{p}(\Sigma)$ which further means that $\hat{h}$ coincides with the degenerate metric $h$ of $T_{p}(\Sigma)$.

Now we denote by $h_{i j}=g_{i j}$ the degenerate induced metric on $\Sigma$ which is the pull back of $g_{i j}$, where an under arrow denotes the pullback to $\Sigma$. $h_{i j}$ has signature $(0,+, \cdots,+)$ and does not have an inverse in the standard sense, but, in the weaker sense it admits an inverse $h^{i j}$ if it satisfies $h_{i k} h_{j m} h^{k m}=h_{i j}$. Then, the expansion $\theta_{(\ell)}$ is defined by $\theta_{(\ell)}=h^{i j} \nabla_{i} \ell_{j}$. The vorticity-free Raychaudhuri equation is given by

$$
\frac{d\left(\theta_{(\ell)}\right)}{d s}=-R_{i j} \ell^{i} \ell^{j}-\sigma_{i j} \sigma^{i j}-\frac{\theta^{2}}{2},
$$

where $\sigma_{i j}={ }_{\leftarrow(i}^{\nabla} \ell_{j)}-\frac{1}{2} \theta_{(\ell)} h_{i j}$ is the shear tensor, $s$ is a pseudo-arc parameter such that $\ell$ is null geodesic and $R_{i j}$ is the Ricci tensor of $M$. Consider a spacelike orthonormal frames field $E=\left\{e_{1}, \cdots, e_{n-2}\right\}$ on $\mathbf{S}_{t}$. As per Proposition 4, the expansion scalar field (null mean curvature) $\theta$ of $\Sigma$ with respect to $\ell$ coincides with the mean curvature of its corresponding spacelike hypersurface $\mathbf{S}_{t}$. Thus, $\theta$ is defined by

$$
\theta=\sum_{\alpha=1}^{n-2} B_{\ell}\left(e_{\alpha}, e_{\alpha}\right)=\sum_{\alpha=1}^{n-2} h\left(\nabla_{\alpha} \ell, e_{\alpha}\right),
$$

which is equivalent with trace $\left(B_{\ell}\right)$ and therefore it does not depend on the frame $E$. For a totally umbilical $\Sigma$ this means that

$$
\theta=\sum_{\alpha=1}^{n-2} B_{\ell}\left(e_{\alpha}, e_{\alpha}\right)=f \sum_{\alpha=1}^{n-2} h\left(e_{\alpha}, e_{\alpha}\right)=(n-2) f .
$$

Then, $\Sigma$ is totally geodesic $(f=0)$ if and only if $\theta$ vanishes, that is, if and only if $\sum_{\alpha=1}^{n-2} B_{\ell}\left(e_{\alpha}, e_{\alpha}\right)=0$.

To prove our first main result (Theorem 6) we need the following form of a second order quasi-linear elliptic operator: Let $\Omega \subset R^{n}$ be connected open sets and $U \subset R^{n} \times R \times R^{n}$. We say that $\mu \in C^{2}(\Omega)$ is $U$-admissible if $\left(x, \mu(x), \partial_{\mu}\right) \in U$ for all $x=\left(x^{1}, \cdots, x^{n}\right) \in U$, where $\partial_{\mu}=\left(\partial_{1} \mu, \cdots, \partial_{n} \mu\right)$ and $\partial_{i} \mu=\partial \mu / \partial x^{i}$. For a $U$-admissible $\mu \in C^{2}(\Omega)$, let

$$
Q=Q(\mu)=\sum_{i, j=1}^{n} a^{i j}(x, \mu, \partial \mu) \partial_{i j} \mu+b(x, \mu, \partial \mu),
$$

where $a^{i j}, b \in C^{1}(U), a^{i j}=a^{j i}$, and $\partial_{i j}=\frac{\partial^{2}}{\partial^{j} \partial^{i}}$. Then, $Q$ is a second order quasi-linear elliptic operator if for each $(x, r, p) \in U$, and $\forall \xi=\left(\xi^{i}, \cdots, \xi^{n}\right) \in R^{n}, \xi \neq 0$,

$$
\sum_{i, j=1}^{n} a^{i j}(x, r, p) \xi^{i} \xi^{j}>0 .
$$

Theorem 5. (Alexandrov, 1954) Let $Q=Q(\mu)$ be a second order quasi-linear elliptic operator. Suppose the $U$-admissible functions $\mu, v \in C^{2}(\Omega)$ satisfy,

(a) $\mu \leq v$ on $\Omega$ and $\mu\left(x_{0}\right)$ for some $x_{0} \in \Omega$, and

(b) $\Omega(v) \leq \Omega(\mu)$ on $\Omega$.

Then, $\mu=v$ on $\Omega$.

Based on above information, we now state and prove the following first main result of this paper:

Theorem 6. Let $\Sigma_{u_{1}}$ and $\Sigma_{u_{2}}$ be two members of a family $F=\left(\left(\Sigma_{u}\right),\left(h_{u}\right)\right)$ of totally umbilical smooth null hypersurfaces of a spacetime $(M, g)$ whose metric $g$ is defined by (2.5). Suppose

(1) $\Sigma_{u_{1}}$ and $\Sigma_{u_{2}}$ meet at $p \in M$ and $\Sigma_{u_{2}}$ lies to the future side of $\Sigma_{u_{1}}$ near $p$

(2) the mean curvatures $\theta_{u_{1}}$ of $\Sigma_{u_{1}}$, and $\theta_{u_{2}}$ of $\Sigma_{u_{2}}$, satisfy, $\theta_{u_{2}} \leq \theta_{u} \leq \theta_{u_{1}}$ for some value of $u$. 
Then $\Sigma_{u_{1}}=\Sigma_{u_{2}}=\Sigma_{u}$ near $p$ and $\theta_{u}$ (which may vanish either on a portion of $\Sigma_{u}$ or on entire $\Sigma_{u}$ ) is the mean curvature of this common null hypersurface $\Sigma_{u}$ for some value of $u$.

In Theorem 2, Galloway proved his maximum principle by taking a prescribed form (suitable for vanishing mean curvature) of a timelike hypersurface $P$ passing through $p$. He took $P$ small enough and considered the spacelike intersection of two null hypersurfaces $\Sigma_{1}$ and $\Sigma_{2}$ with $P$, in a properly transverse manner. Based on this, he used Theorem 5 of strong maximum principle and his hypothesis $\left(\theta_{2} \leq 0 \leq \theta_{1}\right)$ to show that a common null hypersurface $\Sigma_{1}=\Sigma_{2}=\Sigma$ has vanishing mean curvature. Since our working spacetime $(M, g)$ is prescribed in such a way that we have intersecting $H_{t}$ and $\left(\Sigma_{u}\right)$ spacelike and null hypersurfaces of $M$, respectively, and $\left(\mathbf{S}_{t, u}\right)=\left(\Sigma_{u}\right) \cap H_{t}$ common spacelike hypersurfaces (and hence co-dimension two submanifolds of $M$ ), in our approach we do not need a timelike hypersurface of $M$.

Proof. Let $\Sigma \in\left(\Sigma_{u}\right)$ be a null hypersurface of $M$ and $\mathbf{S}_{t} \in\left(\mathbf{S}_{t, u}\right)$ a spacelike hypersurface of $\Sigma$. Suppose $\Sigma$ and $H_{t}$ meet at a point $p \in M$ properly transversely in $\mathbf{S}_{\mathbf{t}}$. Take a spacelike hypersurface $\mathbf{V}_{t}$ of $H_{t}$ passing through $p$ such that $x=\left(x_{1}, \cdots, x_{n-2}\right)$ are its coordinates centred on $p$. Express $\mathbf{S}_{t}$ as a graph over $\mathbf{V}_{t}$, that is, $\mathbf{S}_{t}=$ graph $\mu=\left\{(\mu(x), x) \in \Sigma \cap H_{t}: x \in \mathbf{V}_{t}\right\}$, where $\mu \in C^{\infty}\left(\mathbf{V}_{t}\right)$. Let $Q(\mu)$ be the mean curvature of $\mathbf{S}_{t}=\operatorname{graph} \mu$ and $G$ be the Riemannian metric on $\mathbf{V}_{t}$ whose components are given by $G_{\alpha \beta}(x)=\gamma_{\alpha \beta}(\mu(x), x)$. Then, following first paragraph of this section, it is easy to see that the following expression of $Q(\mu)$ will hold:

$$
Q=Q(\mu)=\sum_{\alpha, \beta=1}^{n-2} a^{\alpha \beta}(x, \mu, \partial \mu) \partial_{\alpha \beta} \mu+b(x, \mu, \partial \mu),
$$

where $\mu$ is a $C^{2} U$-admissible function, $a^{\alpha \beta}, b \in C^{1}(U)$. The operator $Q$ will also satisfy as elliptic operator and, therefore, $Q=Q(\mu)$ is a second order quasi-linear elliptic operator. Take $\ell=(\mathbf{n}+\mathbf{s})$ a future null normal vector field on $\Sigma$. Denote by $B_{H}$ and $B_{\mathrm{s}}$ the second fundamental forms of $H_{t}$ and $\mathbf{S}_{t}$, respectively. Then,

$$
B_{H}(\mathbf{x}, \mathbf{y})=\left\langle\nabla_{\mathbf{x}} \mathbf{n}, \mathbf{y}\right\rangle, \quad B_{\mathbf{s}}(\mathbf{x}, \mathbf{y})=\left\langle\bar{\nabla}_{\mathbf{x}} \mathbf{s}, \mathbf{y}\right\rangle, \quad \forall \mathbf{x}, \mathbf{y} \in T_{q} \mathbf{S}_{t}, \quad q \in \mathbf{S}_{t}
$$

and $\bar{\nabla}$ is an induced metric connection on $\mathbf{S}_{t}$. Then, with respect to an orthonormal basis $\left\{e_{1}, \cdots, e_{n-2}\right\}$ for $T_{q} \mathbf{S}_{\mathbf{t}}$ the value of $\theta$ at $q$ is given by

$$
\begin{aligned}
\theta & =\sum_{\alpha=1}^{n-2} h\left(\nabla_{\alpha} \ell, e_{\alpha}\right)=\sum_{\alpha=1}^{n-2}\left\langle\nabla_{\alpha} \mathbf{n}, e_{\alpha}\right\rangle+\sum_{\alpha=1}^{n-2}\left\langle\nabla_{\alpha} \mathbf{s}, e_{\alpha}\right\rangle \\
& =\sum_{\alpha=1}^{n-2} B_{H}\left(e_{\alpha}, e_{\alpha}\right)+\sum_{\alpha=1}^{n-2} B_{\mathbf{s}}\left(e_{\alpha}, e_{\alpha}\right)=Q_{H}+B_{H}(\mathbf{s}, \mathbf{s})+Q_{\mathbf{s}}
\end{aligned}
$$

Let $\theta(\mu)$ be the null mean curvature of $\Sigma$ along $\mathbf{S}_{t}=$ graph $\mu$. It is straightforward to show that $\theta=\theta(\mu)$ is a second order quasi-linear elliptic operator. Now consider $\Sigma_{u_{1}}$ and $\Sigma_{u_{2}}$ two null hypersurfaces having a common null direction at $p$ and let $H_{t}$ in $M$ pass through $p$ and transverse to this direction. Take $H_{t}$ so small such that the intersections

$$
\mathbf{S}_{t, u_{1}}=\Sigma_{u_{1}} \cap H_{t} \quad \text { and } \quad \mathbf{S}_{t, u_{2}}=\Sigma_{u_{2}} \cap H_{t}
$$

are smooth spacelike hypersurfaces with $\mathbf{S}_{t, u_{2}}$ to the future side of $\mathbf{S}_{t, u_{1}}$ near $p$. As explained above, let $\mathbf{S}_{t, u_{1}}=$ $\operatorname{graph}\left(\mu_{u_{1}}\right), \mathbf{S}_{t, u_{2}}=\operatorname{graph}\left(\mu_{u_{2}}\right)$ and suppose

$$
\theta\left(\mu_{u_{i}}\right)=\left.\theta_{u_{i}}\right|_{\mathbf{S}_{t, u_{i}}=\operatorname{graph}\left(\mu_{u_{i}}\right)}, \quad i=1,2
$$

Taking two normalized null normal vector fields $\ell_{u_{i}}=\left(\mathbf{n}_{u_{i}}+\mathbf{s}_{u_{i}}\right) \in \Gamma\left(T \Sigma_{u_{i}}\right)$, determining $\theta_{u_{1}}$ and $\theta_{u_{2}}$, respectively (as above), a simple computation shows that

$$
\theta\left(\mu_{u_{i}}\right)=Q\left(\mu_{u_{i}}\right)+\text { lower order terms, }
$$

where $Q$ is the mean curvature operator on spacelike graphs over $\mathbf{V}_{t_{u_{i}}}$ in $H_{t}$. The lower order terms involve the second fundamental forms of $H_{t}$ and $\mathbf{S}_{t, u_{i}}$. Thus each $\theta_{u_{i}}$ is a second order quasi-linear elliptic operator. Consequently, using the hypothesis $\theta_{u_{2}} \leq \theta_{u} \leq \theta_{u_{1}}$ we have: 
(1) $\mu_{u_{1}} \leq \mu_{u_{2}}$, and $\mu_{u_{1}}(p)=\mu_{u_{2}}(p)$.

(2) $\theta\left(\mu_{u_{2}}\right) \leq \theta_{u} \leq \theta\left(\mu_{u_{1}}\right)$.

Then the Theorem 5 of Alexandrov's strong maximal principle for second order quasi-linear elliptic PDE's implies that $\mu_{u_{1}}=\mu_{u_{2}}=\mu_{u}$. Thus, $\Sigma_{u_{1}}$ and $\Sigma_{u_{2}}$ agree near $p$. The null normals to $\Sigma_{u_{1}}$ and $\Sigma_{u_{2}}$ in $M$ will then also agree. Therefore, $\Sigma_{u_{1}}=\Sigma_{u_{2}}=\Sigma_{u}$ near $p$ and $\theta_{u}$ (which, as per relation $\theta_{u}=(n-2) f_{u}$ in Equation (3.5), may vanish when $f_{u}$ vanishes on $\Sigma_{u}$ or on its portion) is the mean curvature of this common null hypersurface $\Sigma_{u}$.

Thus, based on our hypothesis $\theta_{u_{2}} \leq \theta_{u} \leq \theta_{u_{1}}$ of Theorem 6, we have proved a geometric maximum principle which obeys mean curvature inequalities of a family of totally umbilical null hypersurfaces of a spacetime manifold which brings in the role of rich geometry of totally umbilical hypersurfaces of a spacetime manifold instead of an earlier restricted work of (Galloway, 2000) on this problem which was only suitable for totally geodesic null hypersurfaces (see Theorem 2). Also, the choice of the metric (2.5) of our working spacetime $(M, g)$ is physically important frame work. For example, the works of Gourgoulhon \& Jaramillo, 2006) on event and isolated horizons and (Duggal, 2012,2014) on time-dependent null horizons used this metric. Moreover, this metric also includes the Robertson-Walker (RW) spacetimes which are very important models both from mathematical and physical (cosmological model) point of view and they further include, among others, the Lorentz-Minkowski spacetime, the Einstein-de-Sitter spacetime, the Friedman cosmological models and the static Einstein spacetime. Thus, our Theorem 6 is applicable to a variety of spacetimes other than only asymptotically flat spacetimes focused in Theorem 2 .

However, Theorem 6 is limited by the fact that not every such totally umbilical null hypersurface can evolve into the vanishing mean curvature totally geodesic null hypersurface which arises, in general relativily, such as black hole event and Cauchy horizon. An example is the family of totally umbilical null cones none of its member can evolve into a totally geodesic hypersurface needed to link it with any black hole null horizon. See details on this example in (Duggal, 2014). Therefore, our analysis is incomplete unless we show that there exists, in particular, a prescribed sub-class of spacetimes for which the mean curvature geometric inequality of the Theorem 6 is valid as well as it establishes a link with Galloway's vanishing mean curvature Theorem 2 for totally geodesic hypersurfaces as models of event and Cauchy horizons. To complete our analysis we need the following induced extrinsic objects. Consider a member $(\Sigma, h, \ell, \mathbf{k})$ of the family $F=\left(\left(\Sigma_{u}\right),\left(h_{u}\right),\left(\ell_{u}\right),\left(\mathbf{k}_{u}\right)\right)$. Using the Equations $(2.7)$ and $(2.8)$, the projector onto $\Sigma$ along $\mathbf{k}$ is defined by

$$
\text { II : } T_{p} M \rightarrow T_{p} \Sigma, \quad \bar{X} \rightarrow X=\bar{X}+g(\ell, \bar{X}) \mathbf{k} .
$$

Above mapping is well defined, i.e., its image is in $T_{p} \Sigma$. Indeed,

$$
\forall \bar{X} \in T_{p} M, \quad g(\ell, \mathbf{I I}(\bar{X}))=g(\ell, \bar{X})+g(\ell, \bar{X}) g(\ell, \mathbf{k})=0 .
$$

Also, $\mathbf{I I}$ is determined only by the foliation of the family $\left(\mathbf{S}_{t, u}\right)$ and not by any rescaling of $\ell$.

Recall that in Chapter 7 of a book by (Duggal \& Jin) a screen distribution was used to obtain induced extrinsic objects of a null hypersurface. Although we are not using any screen, we do have a vector bundle $T \mathbf{S}_{t}$ of the family of $(n-2)$-dimensional hypersurfaces of the family $F$. In order to take from above reference some needed extrinsic structure equations, in this paper we replace the role of screen by the vector bundle $T \mathbf{S}_{t}$ of a member $\Sigma$ of $F$ which has the added advantage that its leaf is obviously integrable. With this understanding, from (2.8) we get the following decomposition.

$$
T M_{\mid \Sigma}=T \Sigma \oplus_{\text {orth }} \operatorname{tr}(T \Sigma)
$$

where $\operatorname{tr}(T \Sigma)=\operatorname{span}\{\mathbf{k}\}$ denotes a null transversal vector bundle of rank 1. Using above decomposition we get the following extrinsic Gauss and Weingarten formulas:

$$
\nabla_{X} Y=\mathcal{D}_{X} Y+B(X, Y) \mathbf{k}, \quad \nabla_{X} k=-A_{\mathbf{k}} X+\tau(X) \mathbf{k}, \quad \forall X, Y \in \Gamma(T \Sigma),
$$

where we denote by $B$ the induced second fundamental form of $\Sigma, A_{\mathbf{k}}$ is called the shape operator on $T_{p} \Sigma, \tau$ and $\mathcal{D}$ are a 1 -form and the induced linear connection $\mathcal{D}$ on $\Sigma$, respectively.

Theorem 7. Let $(M, g)$ be a spacetime with its metric given by (2.5) such that its coordinate time vector $\mathbf{t}=$ $\frac{\partial}{\partial t}=\lambda \mathbf{n}+U$ is a conformal Killing vector $(C K V)$ field. Suppose $F=\left(\left(\Sigma_{u}\right),\left(h_{u}\right),\left(\ell_{u}\right),\left(\mathbf{k}_{u}\right)\right)$ is a family of totally umbilical null hypersurfaces of $(M, g)$ such that the shift spacelike vector field $U$ of its each member $(\Sigma, h)$ is given by $U=\lambda \mathbf{s}-\mathbf{v}$ where $\lambda$ is the lapse function and $\mathbf{v}$ belonging to its correspoding spacelike hypersurface $\left(S_{t}, \hat{h}\right)$ is a Killing vector (CKV) field. Then, 
(a) $£_{\ell} h(X, Y)=\frac{2 \sigma}{\lambda} h(X, Y), \quad \forall X, Y \in T \Sigma$.

(b) $\mathbf{t}$ reduces to a Killing vector field if and only if $\Sigma \in F$ is totally geodesic in $M$.

Proof. By hypothesis we let $\mathfrak{f}_{\mathrm{t}} g=2 \sigma g$ for some conformal function $\sigma$. Then,

$$
\begin{aligned}
& \mathfrak{f}_{\mathfrak{t}} g=2 \sigma g=\langle\mathbf{n}, d \lambda \otimes i d+i d \otimes d \lambda\rangle+\lambda \mathfrak{n}_{\mathbf{n}} g+\mathfrak{£}_{U} g
\end{aligned}
$$

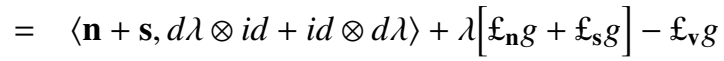

$$
\begin{aligned}
& =\langle\ell, d \lambda \otimes i d+i d \otimes d \lambda\rangle+\lambda\left[\mathfrak{f}_{\mathbf{n}} g+\mathfrak{f}_{\mathbf{s}} g\right] \quad\left(\text { as } \mathfrak{£}_{\mathbf{v}} g=0\right)
\end{aligned}
$$

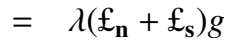

Therefore, projecting both sides of above equation onto $\Sigma$ we get

$$
\lambda\left(£_{\mathbf{n}}+£_{\mathbf{s}}\right) h(X, Y)=2 \sigma h(X, Y), \quad \forall X, Y \in T \Sigma .
$$

On the other hand from (3.8) we know that $\nabla_{X} \ell=\mathcal{D}_{X} \ell$. Using this and computing $£_{\ell} h$ from $\ell=\mathbf{n}+\mathbf{s}$ we obtain

$$
£_{\ell} h(X, Y)=\left(£_{\mathbf{n}}+\mathfrak{f}_{\mathbf{s}}\right) h(X, Y), \quad \forall X, Y \in T \Sigma .
$$

Combining (3.9) and (3.10) proves (a). Then, (b) is immediate since $\sigma=0$ is equivalent to $£_{\ell} h=0$ which implies that $\Sigma \in F$ is totally geodesic in $M$.

Thus, the conclusion (a) implies that the geometric inequality of Theorem 6 is valid if $\mathbf{t}$ is a CKV and $\mathbf{v} \in\left(S_{t}, \hat{h}\right)$ is Killing. Also, we know that each $\Sigma \in F$ is totally geodesic if and only if its mean curvature $\theta$ vanishes. Therefore, the conclusion (b) establishes a link with Galloway's vanishing mean curvature Theorem 2. Consequently, the two Theorems 6 and 7 complete the objective of this paper.

\section{Discussion}

In this paper we have clearly explained that our Theorems 6 and 7 provide an improvement (both on the geometry and its physical use) of a previous work of Galloway's maximum principle (see Theorem 2) which was designed only for vanishing mean curvatuure null hypersurfaces. Also, we claim that this paper is a step forward towards the ongoing physically useful study of time-dependent (non-isolated) null horizons of a variety of spacetimes. To clarify our claim, we first say that since over 50 years the research on null horizons has been limited to timeindependent event and quasi-local isolated horizons. However, in fact the latest research indicates that black holes are surrounded by a local mass distribution and expand by the inflow of galactic debris as well as electromagnetic and gravitational radiations. In other words, the present day spacetime is dynamical and not necessarily stationary. For basic information on latest new development on dynamical spacetimes, we refer (Ashtekar, Beetle \& Fairhurst, 1999) and (Ashtekar \& Krishnan, 2003). Thus, one expects significant difference in the structure and properties of the surrounded time-dependent non-isolated region of the black holes. For this reason, in two recent papers (Duggal, 2012, 2014) a new class of null hypersurfaces of a spacetime $(M, g)$, with metric $(2.5)$, was studied using the following definition:

Definition 8. A null hypersurface $(\Sigma, h, \ell)$ of a spacetime $(M, g)$ is called an Evolving Null Horizon (ENH) if

(i) $\Sigma$ is totally umbilical in $(M, g)$ and may include a totally geodesic portion.

(ii) All equations of motion hold at $\Sigma$ and energy tensor $T_{i j}$ is such that $T_{j}^{i} \ell^{j}$ is future-causal for any future directed null normal $\ell$.

The condition (i) implies from the Proposition 3 that $\mathfrak{f}_{\ell} h=2 f h$ on $\Sigma$, that is, $\ell$ is a conformal Killing vector field of the metric $h$, with conformal function $2 f$. The energy condition of (ii) requires that $R_{i j} \ell^{i} \ell^{j}$ is non-negative for any $\ell$, which implies from page 95 of (Hawking \& Ellis, 1973) that $\theta_{(\ell)}$ monotonically decreases in time along $\ell$, that is, $M$ obeys the null convergence condition, which further means that the null hypersurface $(\Sigma, h)$ is time-dependent in the region where $\theta_{(\ell)}$ is non-zero and may evolve into a time-independent totally geodesic hypersurface as a model of event or isolated horizon. 
Thus, above two implications clearly show that there exists a Model of a class $F=\left(\left(\Sigma_{u}\right),\left(h_{u}\right)\right)$ of a family of totally umbilical null hypersurfaces of $(M, g)$, satisfying the hypothesis of Theorem 6 and Definition 8 , such that its each member is a time-dependent evolving null horizon(ENH).

Now we discuss similarity and difference between our results in this paper and two papers of (Sultana \& Dyer, 2004, 2005) related to common issue of time-dependent null horizons. In their 2004 paper, they considered a conformal transformation $\bar{g}=\Omega^{2} g$ to a stationary, asymptotically flat spacetime $(M, g)$ admitting a Killing horizon $\Sigma$ generated by a Killing field. Since the causal structure and null geodesics are invariant under a conformal transformation, $\Sigma$ still remains a null hypersurface of $(M, \bar{g})$ and its null geodesic generators coincide with the conformal Killing trajectories. They have shown that such a hypersurface $\Sigma$ is null geodesic (they call it conformal Killing horizon) if and only if the twist of the conformal Killing trajectories on $M$ vanish. Moreover, conformal Killing horizons $(\mathrm{CKH})$ are time-dependent null horizons and they can be used to describe locally the event horizon. Then, in their 2005 paper they constructed an example of CKH in the asymptotic background of the de-Sitter universe. Consequently, although their result on existence of time-dependent null $\mathrm{CKH}$ is similar with the two conclusions of our Theorem 7, but, it is only limited to null hypersurfaces of asymptotically flat spacetimes where as our Theorem 7 is applicable to a variety of spacetimes admitting a timelike conformal Killing vector field. This completes the claim that our paper is a step forward towards the ongoing physical use of time-dependent (non-isolated) null horizons of a variety of spacetimes and in some cases their relation with the event and isolated horizons.

\section{References}

Alexandrov, A. D. (1954). Some theorems on partial differential equations of second order, Vestnik Leningrad. Uni. Ser. Mat. Fiz. Him., 9(8), 3-17.

Anderson, L., Galloway, G. J., \& Howard, R. (1998). A strong maximum principle for weak solutions of quasilinear elliptic equations with applications to Lorentzian and Riemannian geometry. Comm. Pure Appl. Math., 51, 581-624. http://dx.doi.org/10.1002/(SICI)1097-0312(199806)51:6;581::AID-CPA2¿3.0.CO;2-3

Arnowitt, R., Deser, S., \& Misner, C. W. (1962). The dynamics of general relativity, in: L. Witten (Ed.), Gravitation: An introduction to current research, Wiley, New York.

Ashtekar, A., Beetle, C., \& Fairhurst, S. (1999). Isolated horizons: a generalization of black hole mechanics, Class. Quantum Grav., 16, L1-L7. http://dx.doi.org/10.1088/0264-9381/16/2/027

Ashtekar, A., \& Krishnan, B. (2003). Dynamical horizons and their properties, Physical Review D, 68, 1403010455. http://dx.doi.org/10.1103/PhysRevD.68.104030

Duggal, K. L. (2012). Foliations of lightlike hypersurfaces and their physical interpretation. Cent. Eur. J. Math., 10(5), 1789-1800. http://dx.doi.org/10.2478/s11533-012-0067-x

Duggal, K. L. (2014). Time-dependent evolving null horizons of a dynamical spacetime. ISRN Mathematical Physics, Article ID 291790, 10 pages, http://dx.doi.org/10.1155/2014/291790.

Duggal, K. L., \& Bejancu, A. (1996). Lightlike Submanifolds of Semi-Riemannian Manifolds and Applications, Kluwer Academic, 364, 1996.

Duggal, K. L., \& Jin, D. H. (2007). Null Curves and Hypersurfaces of Semi-Riemannian Manifolds, World Scientific, 304 pp. http://dx.doi.org/10.1142/6449

Eschenburg, J.-H. (1989). Maximum principle for hypersurfaces, Manuscripta Math., 64, 55-75. http://dx.doi.org/10.1007/BF01182085

Galloway, G. J. (2000). Maximum principles for null hypersurfaces and null splitting theorem, Ann. Henri Poincaré, 1(3), 543-567. http://dx.doi.org/10.1007/s000230050006

Gourgoulhon, E., \& Jaramillo, J. L. (2006). A (1+3)-perspective on null hypersurfaces and isolated horizons, Phys. Rep., 423(4-5), 159-294. http://dx.doi.org/10.1016/j.physrep.2005.10.005

Hawking, S. W. (1972). The event horizons, in Black Holes, edt. C. DeWitt and B. DeWitt, Norht Holland, Amsterdam.

Hawking, S. W., \& Ellis, G. F. R. (1973). The large scale structure of spacetime, Cambridge University Press, Cambridge. http://dx.doi.org/10.1017/CBO9780511524646 
Sultana, J., \& Dyer, C. C. (2004). Conformal Killing horizons, J. Math. Phys., 45(12), 4764-4776. http://dx.doi.org/10.1063/1.1814417

Sultana, J., \& Dyer, C. C. (2005). Cosmological black holes: A black hole in the Einstein-de Sitter universe, Gen. Relativ. Gravit., 37(8), 1349-1370.

\section{Copyrights}

Copyright for this article is retained by the author(s), with first publication rights granted to the journal.

This is an open-access article distributed under the terms and conditions of the Creative Commons Attribution license (http://creativecommons.org/licenses/by/3.0/). 